Vietnam Journal of Mechanics, VAST, Vol. 28, No. 1 (2006), pp. 10-20

\title{
USING FEM FOR LARGE DEFORMATION ANALYSIS OF INFLATED AIR-SPRING CYLINDRICAL SHELL MADE OF RUBBER-TEXTILE CORD COMPOSITE
}

\author{
Tran HuU Nam \\ Hanoi University of Technology
}

\begin{abstract}
An orthotropic hyperelastic constitutive model is presented for large deformation analysis of the nonlinear anisotropic hyperelastic material of the cylindrical air-spring shell used in vibroisolation of driver's seat. Nonlinear hyperelastic constitutive equations of orthotropic composite material are incorporated into the finite strain analysis by finite element method (FEM). The results of deformation analysis of the inflated air-spring shell made of composite with rubber matrix reinforced by textile cords are given. Obtained numerical results of deformation correspond to the experimentally measured deformation of the inflated cylindrical air-spring.
\end{abstract}

Key words: constitutive model, rubber-textile cord composite, air-spring shell

\section{INTRODUCTION}

Nowadays textile composites and inflatable structures are becoming increasingly popular for a variety of applications in many fields - civil engineering, architecture, vehicle and aerospace engineering. Typical examples include membrane roofs and covers, sails, inflatable buildings and pavillions, airships, inflatable furniture, airspace structures, air-springs etc. The composite materials created of rubber matrix reinforced by textile cords is called rubber-textile cord composites ( $R C C$ ). This combination of materials is especially effective not only the composite material needs to be strong in extension in the cord direction, but also needs to be flexible in the plane perpendicular to the cords. This property is the special necessary in cylindrical air-springs which are subjected to air pressure. They need the strength and flexibility in the radial direction.

A phenomenological constitutive model that is capable of predicting the large deformations of composites with rubber matrix has been presented by Nam T.H. (2005). The behavior of the rubber matrix was described by Ogden model and the influence of the cords was embodied by the exponential function that was developed by Holzapfel (2000) and applied to the finite strain calculation of a fiber reinforced rubber tube.

Recently, using FEM to solve the problems of membrane inflation, such as a simplified approach based on the use of Biot stresses in governing equations was proposed by Jiang and Haddow (1995). Their method was successfully applied to the case of initially circular plane membrane. More recently, Shi and Moita (1996) used the finite element (FE) formulation developed previously by Wriggers and Taylor (1990) with the arc-length method to overcome limit points and to calculate secondary branches. Verron et al (2001) presented network-based hyperelastic constitutive equations in the context of thin membrane inflation and proposed a new B-spline interpolation model for the free inflation of axisymmetric rubberlike membrane. The material models are implemented in a numerical tool that incorporates an efficient B-spline interpolation method and a coupled NewtonRaphson/arc-length solving algorithm. Reese et al (2000, 2001) was developed a model 
describing the inelastic material behavior of pneumatic membranes reinforced with rovenwoven textile and modeling finite element for orthotropic material behavior in pneumatic membranes.

In the present paper the orthotropic constitutive model of $\mathrm{RCC}$ is incorporated into the FEM large deformation analysis of the inflated cylindrical air-spring shell. The derivation of the axisymetric shell element kinematics and of the constitutive matrix is presented. The tangent stiffness matrix and the external force vector are also formulated. The computation was carried out in Matlab. Intermediate stages of inflated shell and limit points were computed by the combination of modified Newton-Raphson method with load increments controled by the iteration count of previous convergence and by the arc-length method.

\section{DESCRIPTION OF STRUCTURAL MATERIAL}

A cylindrical air-spring shell is usually made up of four layers - the inner and the outer layer of calandered rubber and the two plies of cord reinforced rubber in which the cords have a specific bias angle to the other arranged symmetrically with respect to the circumferential direction (Fig. 1).

The resulting material properties are orthotropic in the case of in-axis loading. The cylindrical air-spring is relatively short - the diameter of the tubular shell is $2 R=82 \mathrm{~mm}$, the height is $L=120 \mathrm{~mm}$ and the wall thickness is $H=2 \mathrm{~mm}$. The properties of the material and the angle between cords have to be determined experimentally in site since the airspring is assigned for further experiments and it cannot be dissected for usual material tests.
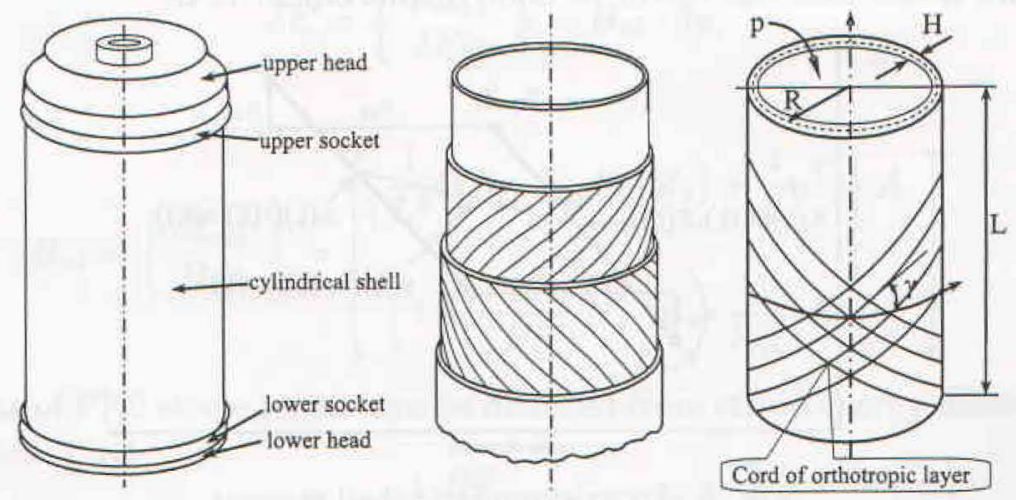

Fig. 1. Textile cord reinforced circular tube of cylindrical air-spring. Continuum model for the structure of the orthotropic layer (with double-helically arranged fibers)

\section{THE ORTHOTROPIC HYPERELASTIC CONSTITUTIVE MODEL}

The orthotropic hyperelastic materials in this paper are considered incompressible composite materials with two families of fibers. Let's assume the isochoric deformation and neglect the dissipation due to irreversible effects. The free energy of the orthotropic hyperelastic materials is considered stored in the matrix material and fibers. Thus, a 
strain energy function which is considered the combination of scalar-value functions corresponding to energy stored in matrix material and the fibers parts in two dimensional problem with incompressibility constraint is

$$
\begin{aligned}
\Psi= & \sum_{a=1}^{3} \frac{\mu_{a}}{\alpha_{a}}\left[\lambda_{1}^{\alpha_{a}}+\lambda_{2}^{\alpha_{a}}+\left(\lambda_{1} \lambda_{2}\right)^{-\alpha_{a}}-3\right]+ \\
& +\frac{k_{1}}{k_{2}}\left\{\exp \left[k_{2}\left(\lambda_{2}^{2} \cos ^{2} \gamma+\lambda_{1}^{2} \sin ^{2} \gamma-1\right)^{2}\right]-1\right\}
\end{aligned}
$$

where $\lambda_{1}, \lambda_{2}$, and $\lambda_{3}$ are the three principal stretches. The parameters $\mu_{a}$ and $\alpha_{a}(\mathrm{a}=1$, $2,3)$ of Ogden's model of rubber (Holzapfel, 2000) are $\mu_{1}=0.63 \mathrm{MPa}, \mu_{2}=0.0012 \mathrm{MPa}$, $\mu_{3}=-0.01 \mathrm{MPa}, \alpha_{1}=1.3, \alpha_{2}=5, \alpha_{3}=-2.2 \gamma$ is the angle of the two families of reinforced fibers, the angle $\gamma$ of fibers is supposed to be $48.8^{\circ}$. The stress-like parameter $k_{1}=41.87$ $\mathrm{MPa}$ and the non-dimensional parameter $\mathrm{k}_{2}=-23.77$ are determined from the experimental measurement and from the 2D cylindrical shell approximation which represented in my previous paper (Nam T.H, 2005).

\section{BASIC EQUATIONS OF NONLINEAR FINITE ELEMENT FORMULATION}

\subsection{The axisymmetric shell element}

The axisymmetric shell element that has length of $L$ and thickness of $H$ in the reference configuration and length of $l$ in the current configuration is presented in Fig. 2. This element has two nodes and the vector of node displacement is $\mathbf{u}$.

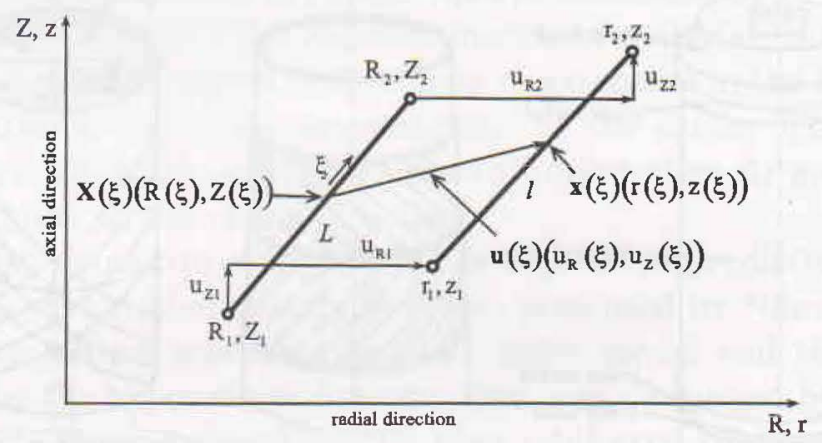

Fig. 2. An axisymmetric shell element

The coordinates in reference and current configuration and displacement vector are interpolated linearly as the functions of the isoparametric coordinate $\xi$

$$
\begin{gathered}
X(\xi)=N \cdot\left\{R_{1}, Z_{1}, R_{2}, Z_{2}\right\}^{T}, \quad x(\xi)=N \cdot\left\{r_{1}, z_{1}, r_{2}, z_{2}\right\}^{T} \\
\boldsymbol{u}(\xi)=\boldsymbol{x}(\xi)-\boldsymbol{X}(\xi)=N \cdot \boldsymbol{u}, \quad \boldsymbol{u}=\left\{u_{R 1}, u_{Z 1}, u_{R 2}, u_{Z 2}\right\}^{T}
\end{gathered}
$$

where $\mathbf{N}$ is the matrix of shape functions

$$
N=\left[\begin{array}{l}
N_{1} \\
N_{2}
\end{array}\right]=\frac{1}{2}\left[\begin{array}{llll}
1-\xi & 0 & 1+\xi & 0 \\
0 & 1-\xi & 0 & 1+\xi
\end{array}\right]
$$


Green-Lagrange (GL) deformation tensor and second Piola-Kirchhoff (PK2) strain rensor are used as the conjugate pair in order to express the strain energy in the Lagrangian description.

The axial Green strain can be found from the change of length element (Shi and Moita, 1996

$$
E_{11}=\frac{l^{2}-L^{2}}{2 L^{2}}=\left[b_{1 l}+b_{1 n}(u)\right] \cdot u,
$$

जhere

$$
\left\{\begin{array}{c}
b_{1 l}=\frac{1}{L^{2}}\left\{R_{1}, Z_{1}, R_{2}, Z_{2}\right\} \cdot A, \\
\boldsymbol{b}_{1 n}(\boldsymbol{u})=\frac{1}{2 L^{2}} \boldsymbol{u}^{T} \cdot \boldsymbol{A},
\end{array} \quad \text { with } \quad \boldsymbol{A}=\left[\begin{array}{cccc}
1 & 0 & -1 & 0 \\
0 & 1 & 0 & -1 \\
-1 & 0 & 1 & 0 \\
0 & -1 & 0 & 1
\end{array}\right]\right.
$$

The hoop component of GL is determined from the change of the circumference length

$$
E_{22}=\frac{o^{2}-O^{2}}{2 O^{2}}=\frac{u_{R}(\xi)}{R(\xi)}+\frac{1}{2}\left(\frac{u_{R}(\xi)}{R(\xi)}\right)^{2}=e_{l}+\frac{1}{2} e_{l}^{2}
$$

where

$$
e_{l}=\frac{1}{R(\xi)} N_{1} \cdot u
$$

The variation $\delta \boldsymbol{E}$ can be expressed by variation $\delta \boldsymbol{u}$ in the following form

$$
\delta \boldsymbol{E}=\left\{\begin{array}{l}
\delta E_{11} \\
\delta E_{22}
\end{array}\right\}=\boldsymbol{B}_{n l} \cdot \delta \boldsymbol{u}
$$

where

$$
\boldsymbol{B}_{n l}=\left[\begin{array}{l}
\boldsymbol{B}_{n l 1} \\
\boldsymbol{B}_{n l 2}
\end{array}\right]=\left[\begin{array}{l}
\frac{1}{L^{2}}\left(\left\{R_{1}, Z_{1}, R_{2}, Z_{2}\right\}+\frac{1}{2} \boldsymbol{u}^{T}\right) \cdot \boldsymbol{A} \\
\left(1+\frac{1}{2 R(\xi)} \boldsymbol{N}_{1} \cdot \boldsymbol{u}\right) \frac{1}{R(\xi)} \boldsymbol{N}_{1}
\end{array}\right]
$$

The components of PK2 stress tensor can be deduced from strain energy function according to the relation

$$
S_{i i}=\frac{1}{\lambda_{i}} \frac{\partial \Psi}{\partial \lambda_{i}}, \quad i=1,2,
$$

where

$$
\begin{gathered}
S_{11}=\sum_{a=1}^{3} \frac{\mu_{a}}{\lambda_{1}^{2}}\left[\lambda_{1}^{\alpha_{a}}-\left(\lambda_{1} \lambda_{2}\right)^{-\alpha_{a}}\right]+4 k_{1} m \exp \left(k_{2} m^{2}\right) \sin ^{2} \gamma \\
S_{22}=\sum_{a=1}^{3} \frac{\mu_{a}}{\lambda_{2}^{2}}\left[\lambda_{2}^{\alpha_{a}}-\left(\lambda_{1} \lambda_{2}\right)^{-\alpha_{a}}\right]+4 k_{1} m \exp \left(k_{2} m^{2}\right) \cos ^{2} \gamma \\
m=\lambda_{2}^{2} \cos ^{2} \gamma+\lambda_{1}^{2} \sin ^{2} \gamma-1 .
\end{gathered}
$$


Furthermore, the components $E_{11}$ a $E_{22}$ of GL can be obtained in the term of principal stretches as

$$
E_{i i}=\frac{\lambda_{i}^{2}-1}{2} \Rightarrow \frac{\partial \lambda_{i}}{\partial E_{i i}}=\frac{1}{\lambda_{i}}, \quad i=1,2 .
$$

The elastic tensor $\mathbb{C}$ is determined from principal components of PK2

$$
\begin{gathered}
\mathbb{C}=\frac{\partial S}{\partial E}, \quad \mathbb{C}=\left[\begin{array}{cc}
\frac{1}{\lambda_{1}} \frac{\partial S_{11}}{\partial \lambda_{1}} & \frac{1}{\lambda_{2}} \frac{\partial S_{11}}{\partial \lambda_{2}} \\
\frac{1}{\lambda_{1}} \frac{\partial S_{22}}{\partial \lambda_{1}} & \frac{1}{\lambda_{2}} \frac{\partial S_{22}}{\partial \lambda_{2}}
\end{array}\right], \quad S=\mathbb{C} E . \\
\mathbb{C}_{11}=\frac{1}{\lambda_{1}^{4}} \sum_{a=1}^{3} \mu_{a}\left[\left(\alpha_{a}-2\right) \lambda_{1}^{\alpha_{a}}+\left(\alpha_{a}+2\right)\left(\lambda_{1} \lambda_{2}\right)^{-\alpha_{a}}\right]+8 k_{1} \exp \left(k_{2} m^{2}\right) \sin ^{4} \gamma\left(1+2 k_{2} m^{2}\right), \\
\mathbb{C}_{12}=\mathrm{C}_{21}=\frac{1}{\lambda_{1}^{2} \lambda_{2}^{2}} \sum_{a=1}^{3} \mu_{a} \alpha_{a}\left(\lambda_{1} \lambda_{2}\right)^{-\alpha_{a}}+8 k_{1} \exp \left(k_{2} m^{2}\right) \sin ^{2} \gamma \cos ^{2} \gamma\left(1+2 k_{2} m^{2}\right), \\
\mathbb{C}_{22}=\frac{1}{\lambda_{2}^{4}} \sum_{a=1}^{3} \mu_{a}\left[\left(\alpha_{a}-2\right) \lambda_{2}^{\alpha_{a}}+\left(\alpha_{a}+2\right)\left(\lambda_{1} \lambda_{2}\right)^{-\alpha_{a}}\right]+8 k_{1} \exp \left(k_{2} m^{2}\right) \cos ^{4} \gamma\left(1+2 k_{2} m^{2}\right)
\end{gathered}
$$

Note that the elasticity tensor is not constant, but depends on the deformations and then must be updated in every iterative step.

\subsection{Principle of virtual work and its linearization}

Principle of virtual work in given problem can be written in the following form:

$$
R(\boldsymbol{u}, \delta \boldsymbol{u}, p)=\int_{\Omega_{0}} S: \delta \boldsymbol{E} d V-p \int_{\partial \Omega} \boldsymbol{n}^{T} \cdot \delta \boldsymbol{u} d s=0,
$$

in which $p$ is the internal pressure, $\delta \mathbf{u}$ stands for a virtual displacement vector, $\Omega_{0}$ is undeformed volume, $\partial \Omega$ represents the deformed shell surface and $\mathbf{n}$ is the normal vector of deformed shell surface.

The principle of virtual work can be expressed through introduction of the external load factor $\lambda$ as

$$
R(\boldsymbol{u}, \delta \boldsymbol{u}, \lambda)=R(\boldsymbol{u}, \lambda)^{T} \cdot \delta \boldsymbol{u}=\left(\boldsymbol{f}_{\text {int }}(\boldsymbol{u})-\lambda \boldsymbol{f}_{\text {ext }}(\boldsymbol{u})\right)^{T} \cdot \delta \boldsymbol{u} .
$$

In this equation $\mathbf{u}$ is the nodal displacement vector $(4.2), \boldsymbol{R}(\boldsymbol{u}, \lambda)$ represents the outof-balance force which must be equal to zero to ensure equilibrium. Moreover $R(\boldsymbol{u}, \lambda)$ can be written under the following form

$$
\boldsymbol{R}(\boldsymbol{u}, \lambda)=\boldsymbol{f}_{\text {int }}(u)-\lambda \boldsymbol{f}_{e x t}(\boldsymbol{u})
$$

The internal force vector of element can be introduced from the first integral of principle of virtual work (4.13) by replacing $\delta \mathbf{E}$ from (4.7) and $\mathrm{dV}=2 \pi R(\xi) H L d \xi$

$$
f_{i n t}^{e}=\int_{-1}^{1} B_{n l}^{T} S 2 \pi R(\xi) H L d \xi
$$


Similarly the external force vector of element can be introduced from the second integral of principle of virtual work (4.13) by replacing $\mathrm{d} s=2 \pi r(\xi) l d \xi$

$$
f_{\text {ext }}^{e}=\int_{-1}^{1} \boldsymbol{N}^{T} \cdot \boldsymbol{n}(\xi) 2 \pi r(\xi) l d \xi
$$

where $\boldsymbol{n}(\xi)=\frac{1}{l}\left\{\begin{array}{l}z_{2}-z_{1} \\ -\left(r_{2}-r_{1}\right)\end{array}\right\}, r(\xi)=\boldsymbol{N}_{1} \cdot\left\{r_{1}, z_{1}, r_{2}, z_{2}\right\}^{T}$.

Now a system of nonlinear equation is obtained in the following form

$$
f_{\text {int }}(u)-\lambda f_{\text {ext }}(u)=0,
$$

In order to solve the nonlinear equations (4.18), the classical tangent stiffness matrix $\boldsymbol{K}_{t}=\frac{\partial \boldsymbol{R}(\boldsymbol{u}, \lambda)}{\partial \boldsymbol{u}}=\left[\boldsymbol{K}_{\text {int }}-\boldsymbol{K}_{\text {ext }}\right]$ has to be defined (Chevaugeon et al, 2002). The stiffness matrix $\mathbf{K}_{t}$ is expressed in the following form

$$
\boldsymbol{K}_{t}=\left[\frac{\partial \boldsymbol{f}_{\text {int }}}{\partial \boldsymbol{u}}-\lambda \frac{\partial \boldsymbol{f}_{\text {ext }}}{\partial \boldsymbol{u}}\right] .
$$

By the derivative of internal force vector (4.16) with respect to the displacements, it follows that

$$
\begin{gathered}
\frac{\partial \boldsymbol{f}_{i n t}^{e}}{\partial \boldsymbol{u}}=\boldsymbol{K}_{M}^{e}+\boldsymbol{K}_{G}^{e} \\
\boldsymbol{K}_{M}^{e}=\int_{-1}^{1} \boldsymbol{B}_{n l}^{T} \mathbb{C} \boldsymbol{B}_{n l} 2 \pi R(\xi) H L d \xi \\
\boldsymbol{K}_{G}^{e}=\int_{-1}^{1}\left(\frac{S_{11}}{2 L^{2}} \boldsymbol{A}+\frac{S_{22}}{R^{2}(\xi)} \boldsymbol{N}_{1}^{T} \boldsymbol{N}_{1}\right) 2 \pi R(\xi) H L d \xi
\end{gathered}
$$

in which $\boldsymbol{K}_{M}^{e}$ is the standard stiffness matrix or initial displacement matrix and $\boldsymbol{K}_{G}^{e}$ is the geometric or initial stress stiffness matrix.

Similarly by the derivative of external force vector (4.17) with respect to the displacements will be obtained the following matrix

$$
\begin{gathered}
\boldsymbol{K}_{p}^{e}=\frac{\partial \boldsymbol{f}_{e x t}^{e}}{\partial \boldsymbol{u}}=\frac{2 \pi}{3}\left[\begin{array}{llll}
2 z_{21} & -\left(2 r_{1}+r_{2}\right) & z_{21} & 2 r_{1}+r_{2} \\
4 r_{1}-r_{2} & 0 & -\left(r_{1}+2 r_{2}\right) & 0 \\
z_{21} & -\left(r_{1}+2 r_{2}\right) & 2 z_{21} & r_{1}+2 r_{2} \\
2 r_{1}+r_{2} & 0 & r_{1}-4 r_{2} & 0
\end{array}\right], \\
z_{21}=z_{2}-z_{2}, \quad\left\{r_{1}, z_{1}, r_{2}, z_{2}\right\}=\left\{R_{1}, Z_{1}, R_{2}, Z_{2}\right\}+\left\{u_{R 1}, u_{Z 1}, u_{R 2}, u_{Z 2}\right\}
\end{gathered} .
$$

The total stiffness matrix for one element is determined by the initial displacement matrix $\boldsymbol{K}_{M}^{e}$, the initial stress matrix $\boldsymbol{K}_{G}^{e}$ and the crucial load tangent stiffness matrix caused deformation dependent loads (Holzapfel et al, 1996), as:

$$
\boldsymbol{K}_{t}^{e}=\boldsymbol{K}_{M}^{e}+\boldsymbol{K}_{G}^{e}-\lambda_{n} \boldsymbol{K}_{p}^{e}
$$




\section{NONLINEAR NUMERICAL SOLUTION}

The basic approach to solve the nonlinear responses is the incremental-iterative method or the continuation method, also called path-following method determining the equilibrium points on the load (pressure) -displacement paths or equilibrium paths. The incremental-iterative approach described here is based on a combination of the modified Newton - Raphson iterative method and the arc-length method. The tangent stiffness matrix and internal force vector and external force vector are updated not only at the commencement of every iterative cycle, but also at each load step (Fig. 3).

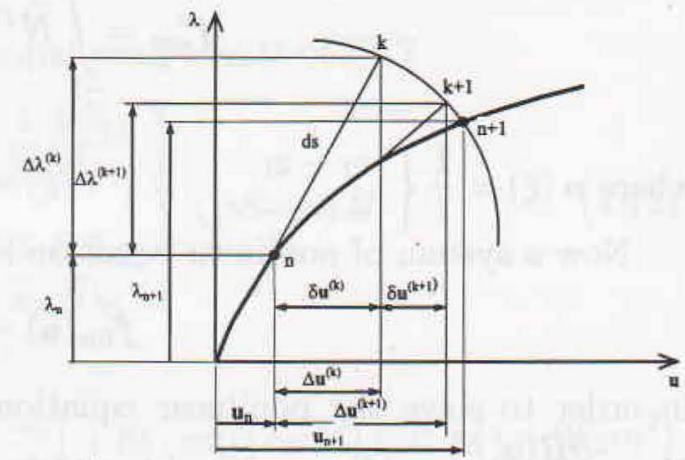

Fig. 3. The combination of modified Newton-Raphson and arc-length method

\subsection{Predictor and corrector solutions}

Consider a particular equilibrium point at an instant $t_{n}$ on the equilibrium path which is defined by the nodal displacement $\boldsymbol{u}_{n}$ and load factor $\lambda_{n}$. The purpose of the numerical solution is to find one new equilibrium point on the path. The new point at subsequent instant $t_{n+1}$ is defined by displacement and load factor increments denoted $\Delta u$ and $\Delta \lambda$, respectively, and it satisfies simultaneously the following equations

$$
\left\{\begin{array}{l}
\boldsymbol{R}\left(\boldsymbol{u}_{n}+\Delta \boldsymbol{u}, \lambda_{n}+\Delta \lambda\right)=0 \\
F(\Delta \boldsymbol{u}, \Delta \lambda)=0
\end{array}\right.
$$

The first equation is the residual equation and the second one is extra equation which is normally called arc-length constraint associated with path-following procedures. For the more widely used cylindrical arc-length method, the constraint equation is given in the following form (Crisfield, 1997; De Souza Neto and Feng, 1999)

$$
\Delta \boldsymbol{u}^{T} \Delta \boldsymbol{u}=d s^{2},
$$

where ds is arc-length which is the control parameter.

Here the Newton-Raphson iterative method is applied to the previous system (5.1) is now examined. Consider the algorithm at a given iteration, the system to be solved is

$$
\left\{\begin{array}{l}
R^{(k+1)}=R^{(k)}+\frac{\partial R}{\partial \hat{A} u} \delta u+\frac{\partial R}{\partial \hat{A} \lambda} \delta \lambda=0 \\
F^{(k+1)}=F^{(k)}+\frac{\partial F^{\prime}}{\partial u} \delta u+\frac{\partial F}{\partial \Delta \lambda} \delta \lambda=0
\end{array}\right.
$$

where subscripts $k+1$ and $k$, respectively, are for the current iteration and for the previous iteration. For the solution displacement vector is obtained as

$$
\boldsymbol{u}_{n+1}^{(k+1)}=\boldsymbol{u}_{n}^{(k)}+\Delta \boldsymbol{u}^{(k+1)},
$$




$$
\Delta \boldsymbol{u}^{(k+1)}=\Delta \boldsymbol{u}^{(k)}+\delta \boldsymbol{u}^{(k+1)},
$$

where $\Delta \boldsymbol{u}^{(k)}$ and $\Delta \boldsymbol{u}^{(k+1)}$ are the incremental displacement guesses for the previous and crrent iteration, respectively, and $\delta \boldsymbol{u}$ is the iterative displacement. The incremental load izctor, $\Delta \lambda$, is updated according to

$$
\Delta \lambda^{(k+1)}=\Delta \lambda^{(k)}+\delta \lambda .
$$

U $=$ ing the previous definition of the tangent stiffness matrix (4.19) the system (5.3) becomes

$$
\left\{\begin{array}{l}
\boldsymbol{R}^{(k+1)}=\boldsymbol{R}^{(k)}+\boldsymbol{K}_{t}\left(\boldsymbol{u}^{(k)}\right) \delta u-\delta \lambda \boldsymbol{f}_{\text {ext }}\left(\boldsymbol{u}^{(k)}\right)=0, \\
F^{(k+1)}=F^{(k)}+2 \Delta \boldsymbol{u}^{(k)} \cdot \delta \boldsymbol{u}=0
\end{array}\right.
$$

The system of nonlinear equations for iterative solutions $\delta u$ and $\delta \lambda$ (the corrector solution) is derived by simply linearising the residual equation and cylindrical arc-length equation using Taylor series. The system of equations (5.7) is written as

$$
\left[\begin{array}{ll}
\boldsymbol{K}_{t}\left(u^{(k)}\right) & -f_{\text {ext }}\left(\boldsymbol{u}^{(k)}\right) \\
2 \Delta \boldsymbol{u}^{(k) T} & 0
\end{array}\right]\left\{\begin{array}{l}
\delta \boldsymbol{u} \\
\delta \lambda
\end{array}\right\}=-\left\{\begin{array}{l}
R\left(\boldsymbol{u}^{(k)}, \Delta \lambda^{(k)}\right) \\
\Delta \boldsymbol{u}^{T} \Delta \boldsymbol{u}-d s^{2}
\end{array}\right\}
$$

where the subscript $n+1$ have been abandoned for notational convenience.

When $k=0$ (the predictor solution), the forward-Euler tangential predictor solution is adopted (Verron and Marckmann, 2001). The two predicted increments $\Delta \bar{u}$ and $\Delta \bar{\lambda}$ are supposed to satisfy the first equilibrium equation of $(5.7)$ with $R^{(0)}=0$

$$
\Delta \overline{\boldsymbol{u}}=\Delta \bar{\lambda} \delta \bar{u},
$$

where tangent displacement vector $\delta \bar{u}$ is given by

$$
\delta \bar{u}=K_{t}^{-1} f_{\text {ext }} .
$$

The possible iterative load factor $\delta \lambda$ for the predictor solution is

$$
\delta \lambda= \pm \frac{d s}{\sqrt{\delta \overline{\boldsymbol{u}}^{T} \delta \bar{u}}}
$$

and the success of the path-following technique depends crucially on the choice of the appropriate sign for iterative load factor.

In order to predict the continuation direction the sign of predictor load factor must be chossen. From the criterion of Feng (Feng Y.T et al, 1996) the sign of the predictor load factor is made to coincide with the sign of the internal product between the previous converged incremental displacement $\Delta \boldsymbol{u}_{n}$, and the current tangential solution $\delta \bar{u}$.

$$
\operatorname{sign}(\delta \lambda)=\operatorname{sign}\left(\Delta \boldsymbol{u}_{n}^{T} \delta \bar{u}\right)
$$

\subsection{Step-length control}

For controlling the step length size the arc-length for use in the current increment $(n+1)$ can be computed using the arc-length of previous increment $(n)$ by

$$
d s_{n+1}=d s_{n}\left(\frac{J_{d}}{J_{n}}\right)^{\eta}
$$


in which $d s_{n}$ is arc-length for previous load step $n, J_{n}$ is actual number of iterations required for convergence in the previous load step, $J_{d}$ is a user-defined desired number of iterations for convergence, typically 3 to 5 . The exponent $\eta$ usually lies in the range 0.5 to 1.0 .

\subsection{Convergence Criterion}

A some convergence criterion based on the incremental displacements or energy are presented (Clarke and Hancock, 1990). The residual convergence criterion is introduced here. The Euclidean norm of residual is compared with some predefined tolerance:

$$
\|\boldsymbol{R}(\boldsymbol{u}, \lambda)\| \leq \varepsilon_{r},
$$

where typically $\varepsilon_{r}$ is in the range $10^{-2}$ to $10^{-5}$, depending on the desired accuracy and the non-linear charateristics of the particular problem.

\section{NUMERICAL RESULTS}

In this section the inflation of a cylindrical CRC membrane of air-spring shell is examined. The problem 2D geometry and the boundary condition are shown in Fig. 4 . The material of air-spring considerred is the orthotropic hyperelastic material and modelled by constitutive equations through the strain energy function (3.1). The material parameters which were determined previously are used in FEM computation. Because of symmetrical distribution the half of air-spring shell was discretized by 60 elements. In the first phase the calculation was implemented by selecting small incremental force and in every iterative step iterations were performed until achievement the required toleration. The combination of modified Newton-Raphson method and arc-length procedure was used in the next phase in which the stiffness matrix, internal and external force vector were updated not only in the beginning of every loading step, but also in every iterative cycle (Fig. 3).

Numerical results are obtained after simulating the inflation of cylindrical shell by predictor-corrector method using my program script files written in Matlab. Fig.5 describes equilibrium path between radial displacement with internal pressure. The equilibrium path is obtained up to overcome the limit point and corresponds with the experimental responses.

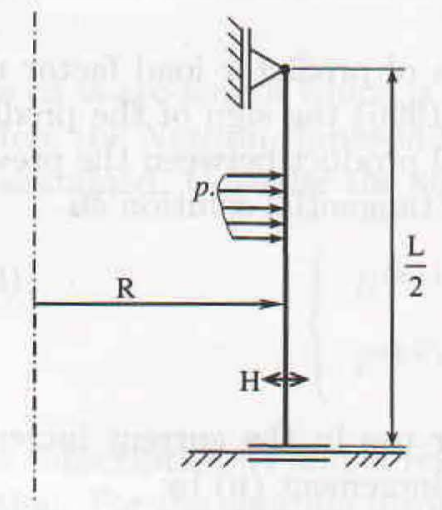

Fig. 4. FEM model of inflated cylindrical shell with free heads

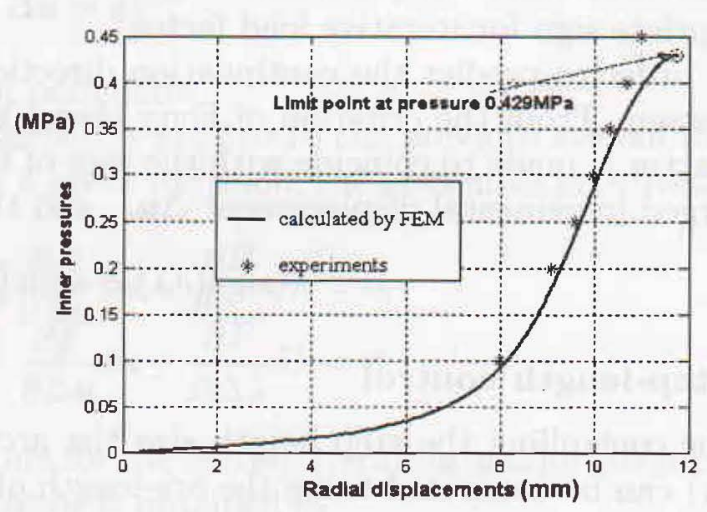

Fig. 5. Equilibrium paths in the case of free heads 
T: proties of deformed shell at different stages of loading are described in Fig. 6.

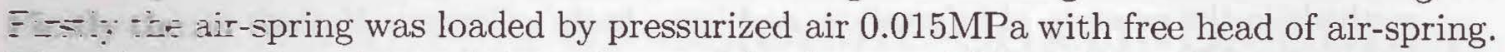
T- $=-Z=$ mounting plates of air-spring were fixed meanwhile the internal presure comes up - our. IPa. Fig. 7 describes the deformed shape of the cylindrical air-spring shell. Fig. $\therefore=\Sigma i z$. 7 show that the ends of examined air-spring influence greatly its deformation. .

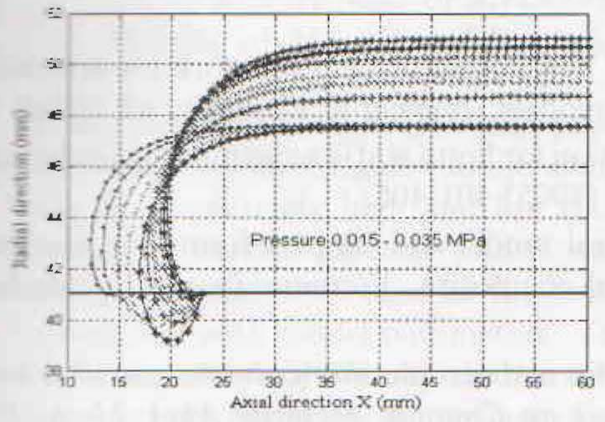

Fig. 6. Profiles of deformed shell at different stages of loading

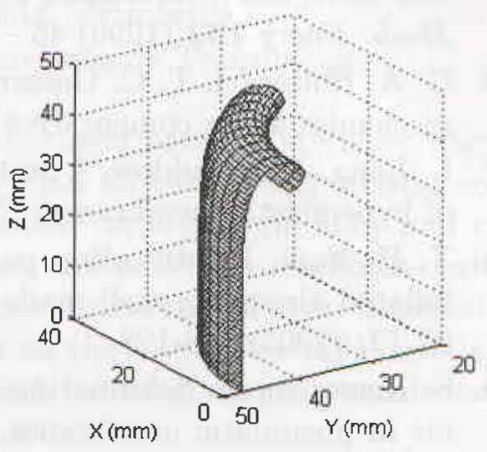

Fig. 7. Deformed shape under internal pressure $p=0.015 \mathrm{MPa}$

It is evident from the figures above once the heads of the air-spring approach together the deformed shapes become curved broadly near the heads of air-spring.

\section{CONCLUSIONS}

The nonlinear FE formulation for the finite strain behavior has been derived and verified. The strain energy function (3.1) was used to set up the constitutive relations in the finite element analysis using Matlab code. Axisymmetric finite elements for the hyperelastic orthotropic material model were made up to simulate the deformation of an inflated cylindrical membrane. The highly nonlinear numerical procedure was mastered. Limit points were detected in the equilibrium following path by using combination of modified Newton-Raphson and arc-length methods in FE analysis. The developed finite elements are efficient and, in general, applicable for various materials in the large strain range. The performance of the both developed softwares and the accuracy of the numerical results have been demonstrated for several examples in which the obtained numerical results answered to experimental responses.

Acknowledgement. This publication is completed with financial support from the $\mathrm{Na}$ tional Basic Research Program in Natural Science and was realized in the framework of the project science research of Hanoi University of Technology 2006.

\section{REFERENCES}

1. N. Chevaugeon, G. Marckmann, E. Verron, B. Peseux, Instabilité et Bifurcation du Souffage de Membranes Hyperélastiques, REEF, 2002, pp $479-492$.

2. M. J. Clarke, G. J. Hancock, A study of incremental-iterative strategies for nonlinear analyses, Int. J. for Numerical Methods in Engrg. 29 (1990) 1365-1391. 